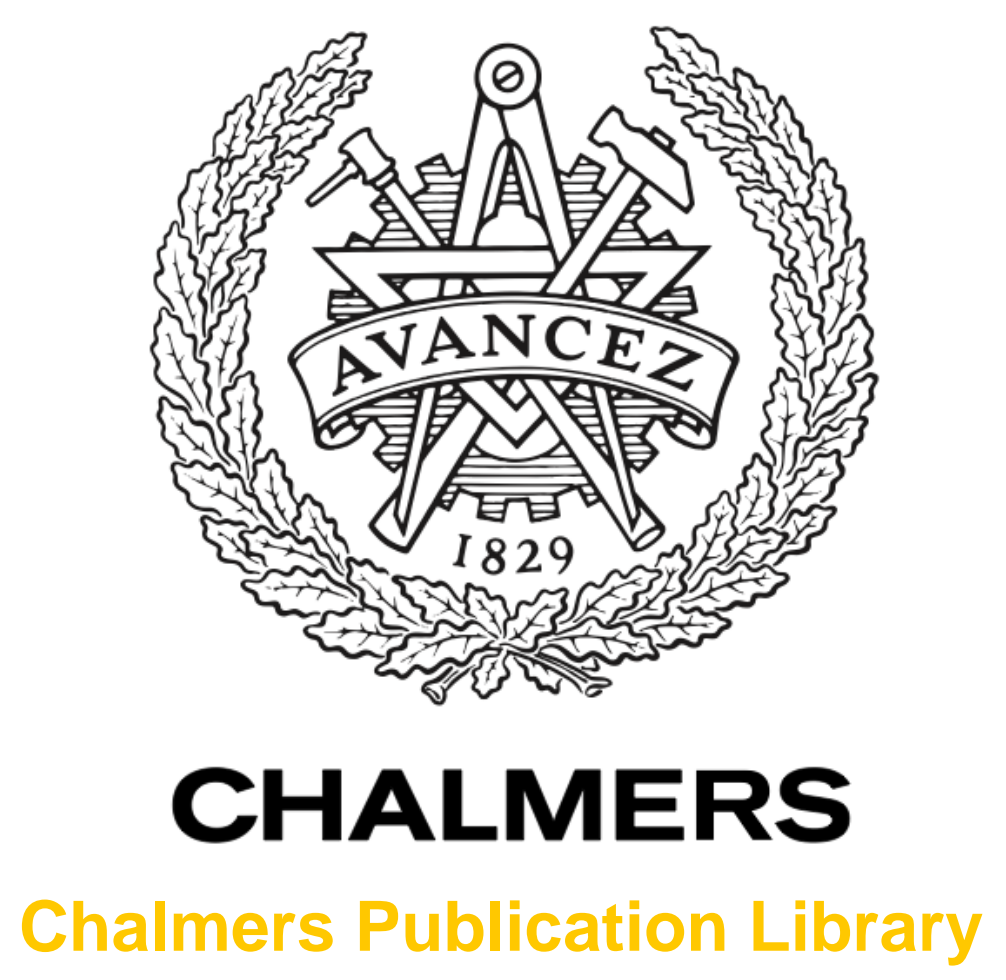

\title{
Covalent functionalization of carbon nanotube forests grown in situ on a metal- silicon chip
}

This document has been downloaded from Chalmers Publication Library (CPL). It is the author's version of a work that was accepted for publication in:

Proceedings of SPIE - The International Society for Optical Engineering. Nanosensors, Biosensors, and Info-Tech Sensors and Systems 2012, San Diego, CA, 12-15 March 2012 (ISSN: 0277-786X)

Citation for the published paper:

Johansson, J. ; Bosaeus, N. ; Kann, N. (2012) "Covalent functionalization of carbon nanotube forests grown in situ on a metal-silicon chip". Proceedings of SPIE - The International Society for Optical Engineering. Nanosensors, Biosensors, and Info-Tech Sensors and Systems 2012, San Diego, CA, 12-15 March 2012, vol. 8344

http://dx.doi.org/10.1117/12.915397

Downloaded from: http://publications.lib.chalmers.se/publication/158220

Notice: Changes introduced as a result of publishing processes such as copy-editing and formatting may not be reflected in this document. For a definitive version of this work, please refer to the published source. Please note that access to the published version might require a subscription. 


\title{
Covalent Functionalization of Carbon Nanotube Forests Grown In Situ on a Metal-Silicon Chip
}

\author{
Johan R. Johansson ${ }^{\mathrm{a}}$, Niklas Bosaeus ${ }^{\mathrm{a}}$, Nina Kann ${ }^{\mathrm{a}}$, Björn Åkerman ${ }^{\mathrm{a}}$, Bengt Nordén ${ }^{\mathrm{a}}$ and Waqas \\ Khalid*a,b,c $^{*}$ \\ ${ }^{a}$ Department of Chemical and Biological Engineering, Chalmers University of Technology, SE- \\ 41296 Gothenburg, Sweden \\ bJadoo Technologies Inc, Toronto, ON, Canada \\ cLawrence Berkeley National Labs \& Chemistry Department, UC Berkeley, Berkeley, CA, USA \\ 94720 \\ *dr.waqaskhalid@gmail.com or wkhalid@lbl.gov; phone 1313-333-3551
}

\begin{abstract}
We report on the successful covalent functionalization of carbon nanotube (CNT) forests, in situ grown on a silicon chip with thin metal contact film as the buffer layer between the CNT forests and the substrate. The CNT forests were successfully functionalized with active amine and azide groups, which can be used for further chemical reactions. The morphology of the CNT forests was maintained after the functionalization. We thus provide a promising foundation for a miniaturized biosensor arrays system that can be easily integrated with Complementary Metal-Oxide Semiconductor (CMOS) technology.
\end{abstract}

Keywords: covalent functionalization, carbon nanotubes, functionalized CNTs on a chip, SEM, EDS, 1,3-cycloaddition, CuAAC, amide coupling

\section{INTRODUCTION}

Carbon nanotube forests have been the focus of interest for research in various areas of science and technology including in the context of design of microelectronics, super capacitors and biosensors. ${ }^{1-4}$ The unique electrical, mechanical, optical and thermal properties of CNT forests are key factors that make such CNT structures interesting candidates for numerous nanotechnology applications. Various efforts to functionalize CNTs chemically have been reported in the literature where the CNTs are derivatized by covalent and non-covalent attachment of functional groups. ${ }^{5-8}$ Conventional methods usually employ techniques to functionalize the CNTs when dispersed in solution and the CNTs are redeposited on surfaces after the modification. Generally the effort to chemically modify the CNTs is carried out in order to enhance the solubility of the CNTs in various solvents, ${ }^{9}$ and harsh oxidizing agents like nitric acid or strong bases are frequently used. Such techniques cannot be employed to functionalize CNTs when on chip, as the underlying metal films are attacked by the reagents. The dispersive technique leads to larger process errors and issues with reproducibility of devices. Hence, there is a strong desire to find a method to functionalize CNT structures as grown on chip. This enhances

Nanosensors, Biosensors, and Info-Tech Sensors and Systems 2012, edited by Vijay K. Varadan, Proc. of SPIE Vol. 8344, 834411 · @ 2012 SPIE · CCC code: 0277-786X/12/\$18 · doi: 10.1117/12.915397 
the reproducibility in the fabrication process of any devices. Also, the use of such structures as sensors is much simpler, more economical and CMOS compatible when they are functionalized in situ grown on chips using reactions at relatively low temperatures and atmospheric pressure, and without any aggressive chemicals. Moreover, the underlying metal films that are used to ensure good electrical contact between all the CNTs in a CNT forest are protected from damage. Thus, the combination of the electrical properties of carbon nanotube forests, their morphology and the ease of functionalization can offer novel ways of immobilizing biomolecules on extremely large surface areas of these structures, providing a potential platform for making ultra-sensitive biosensors. Herein we report a successful and relatively mild covalent functionalization process of CNTs in situ grown on a chip.

\section{METHODOLOGY}

\subsection{Fabrication process}

The fabrication process for the CNT forest on chip is described in Scheme 1.

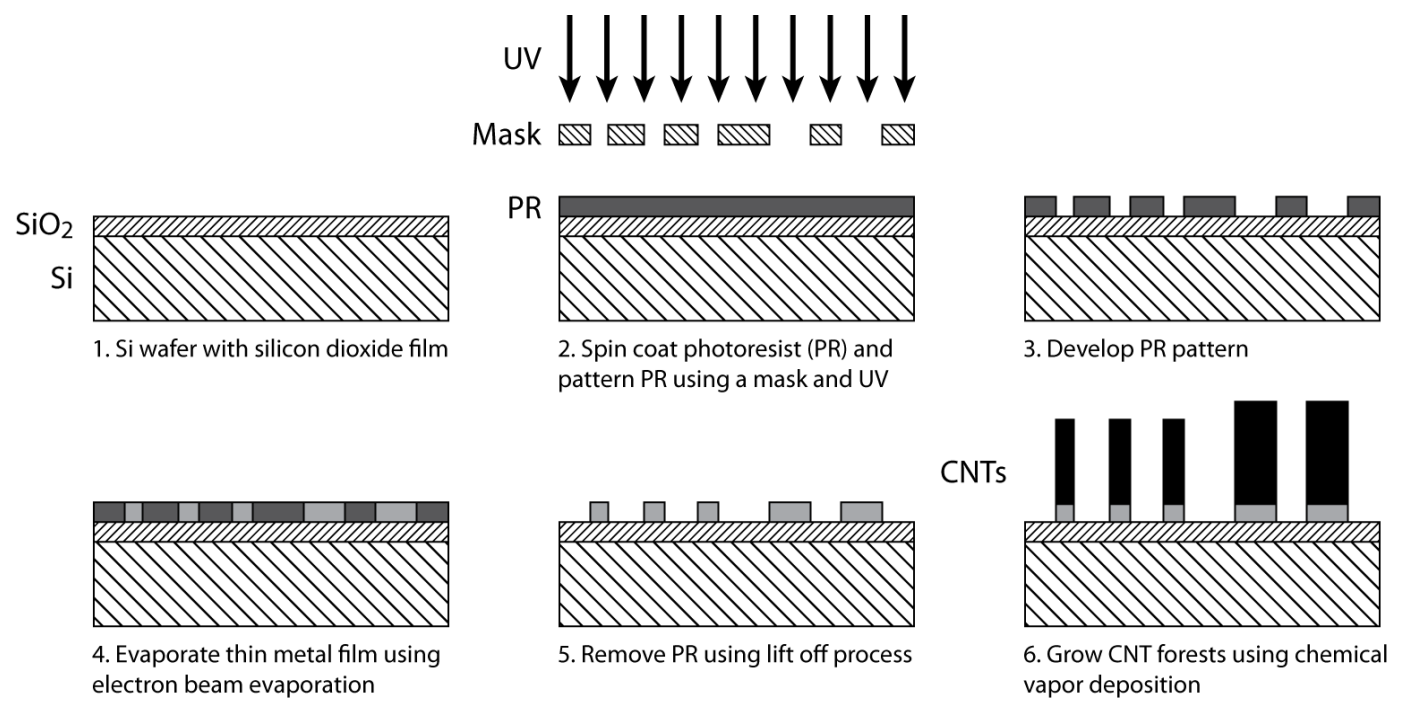

Scheme 1: The fabrication process of CNT forest structures.

A silicon wafer with a $0.5 \mu \mathrm{m}$ silicon oxide was used as the substrate. Using lithography, a pattern was created on a photo resist (PR) film on the substrate. Electron beam evaporation was employed to deposit thin films of metals (Ti 1 $\mathrm{nm} / \mathrm{Mo} 100 \mathrm{~nm} / \mathrm{Al}_{2} \mathrm{O}_{3} 5 \mathrm{~nm} / \mathrm{Fe} 1 \mathrm{~nm}$ ). An Iron film was used as a catalyst to grow the CNT forests. After e-beam evaporation, the photoresist was dissolved in an organic solvent (photoresist remover) assisted by an ultrasonic bath, a process known as lift-off. The wafer was diced into chips and CNT forests were grown on the chips using a thermal chemical vapor deposition (CVD) process. In this process, the catalyst (iron) was first annealed at $500{ }^{\circ} \mathrm{C}$ for 5 minutes in the presence of hydrogen and nitrogen. Later, ethylene, hydrogen and nitrogen gases were added at $700{ }^{\circ} \mathrm{C}$, and $\mathrm{CNT}$ forests were grown by CVD. The process was carried out in a cold tube furnace system (Black Magic by Aixtron). The resistance between the tips of the CNT structures range from $100-200 \Omega$. A picture and a schematic drawing of the CNT forests on chip produced are shown in Figure 1. 

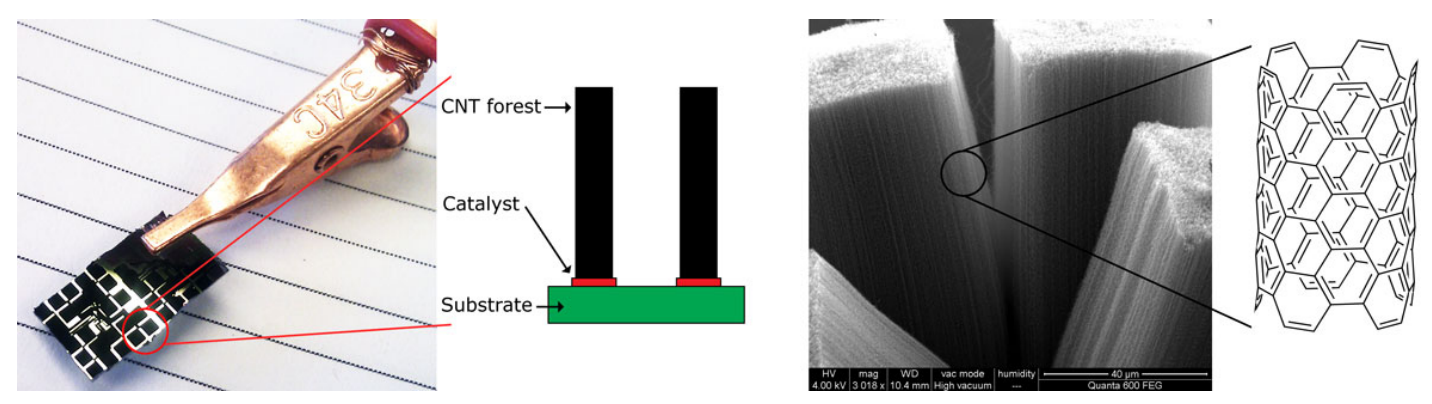

Figure 1. The black squares in the picture (left) are CNT forests and the schematic drawing (middle) shows how the CNT forests are bound to the surface on chip. A SEM micrograph of CNT forest consists of a bundle of many vertically aligned CNTs (right).

\subsection{Functionalization process}

Our strategy to functionalize CNT forests on chip is based on the well known 1,3 dipolar cycloaddition of azomethine ylides to activated double bonds, which have been applied to both $\mathrm{C}_{60}{ }^{10}$ and $\mathrm{SWCNT}^{11}$ in solution and is outlined in Scheme 2. The azomethine ylide was formed in situ upon heating of glycine and benzaldehyde to $120{ }^{\circ} \mathrm{C}$ in dichlorobenzene and reacted with double bonds in the CNT structures on chip (Figure 2). The procedure yielded the amine-modified CNT forest on chip, $f$-CNT 1, after cleaning. $f$-CNT 1 was further functionalized by employing an amide coupling with $O$-(2-azidoethyl)- $O$-[2-(diglycolyl-amino)ethyl]heptaethylene glycol to introduce azide functionalities on the CNTs. The amide coupling was carried out using $\mathrm{PyBOP}^{12}$ as the coupling reagent under mild basic conditions (DIPEA in anhydrous $\mathrm{CH}_{2} \mathrm{Cl}_{2}$ at ambient temperature) to obtain azide functionalized CNT forests on chip, $f$-CNT 2. The functionalized CNT forests on chip were analyzed using SEM equipped with EDS.

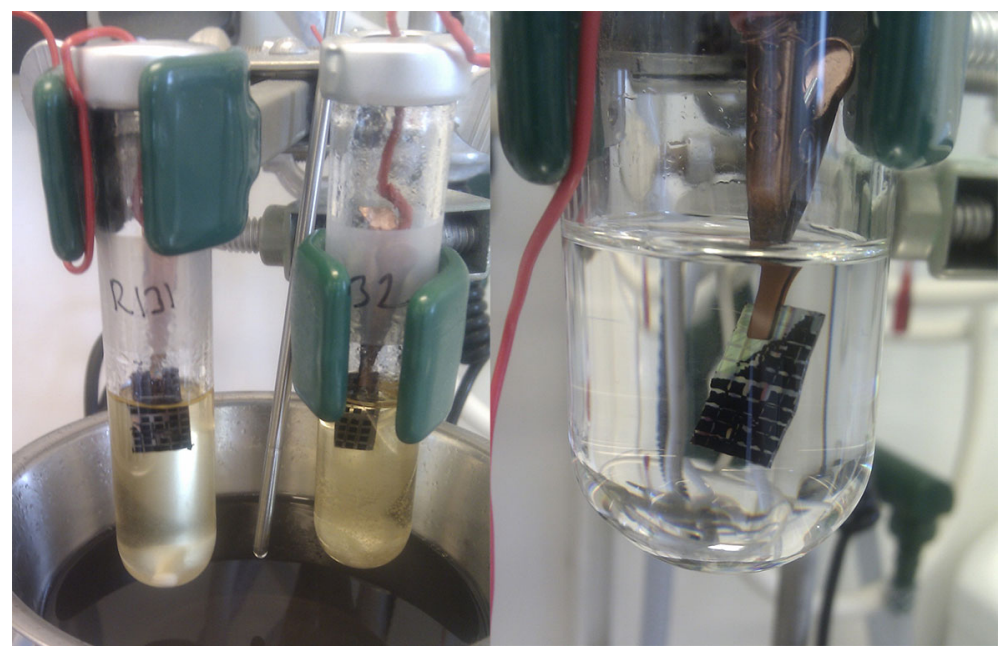

Figure 2. Left picture shows the reaction setup (after reaction) and right picture shows the cleaning setup, performed three times for a minimum of $1 \mathrm{~h}$ after any reaction step. 
To prove that $f$-CNT 2 were functionalized with azide groups, fluorine was introduced via a CuAAC reaction ${ }^{13-14}$ with 1ethynyl-4-(trifluoromethyl)benzene at ambient temperature to obtain $f$-CNT 3. As a control experiment, $f$-CNT 1 was also treated with 1-ethynyl-4-(trifluoromethyl)benzene under similar reaction conditions and analyzed by SEM equipped with EDS.
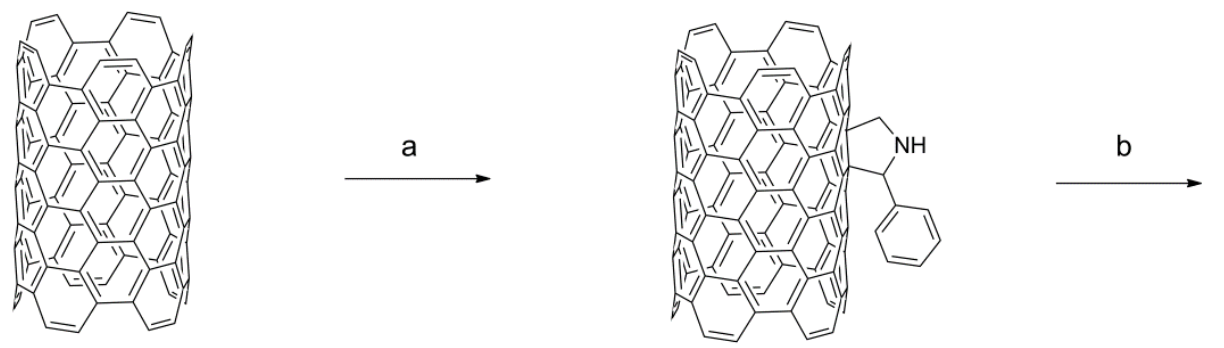

$f$-CNT 1

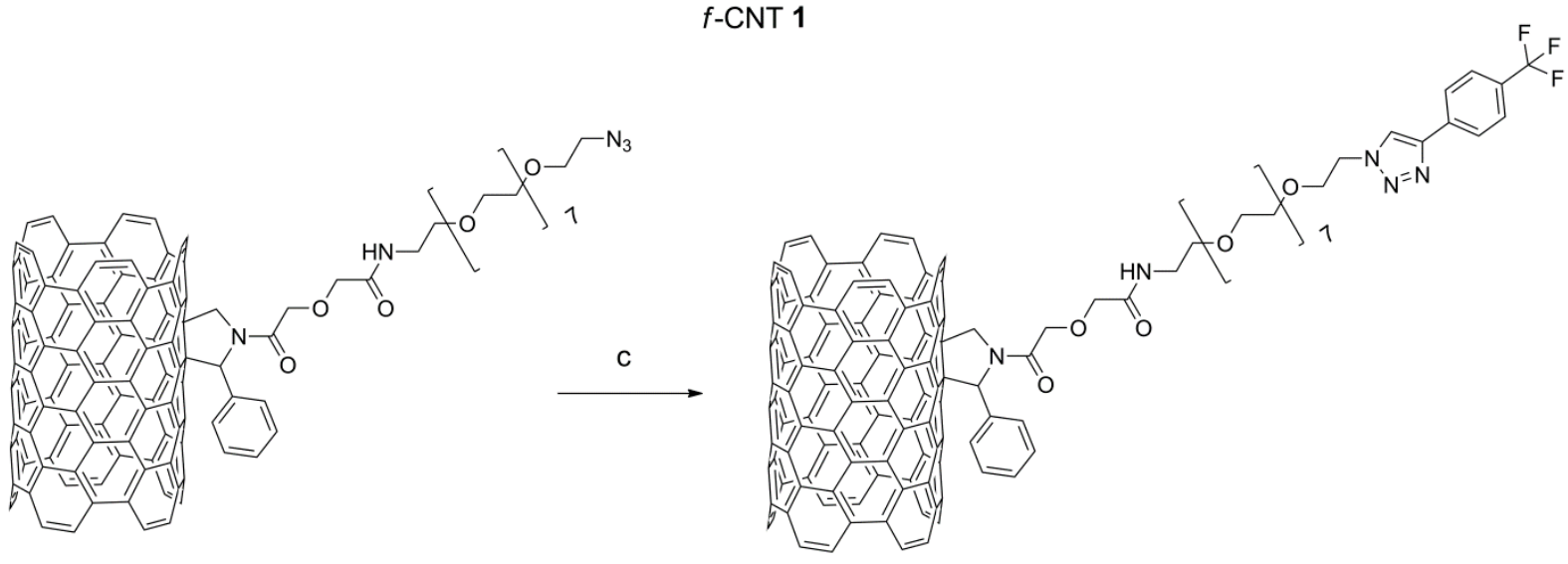

$f$-CNT 2

$f$-CNT 3

Scheme 2. (a) Glycine, benzaldehyde, dichlorobenzene, $120^{\circ} \mathrm{C}$ for 6 to 48 h. (b) PyBOP, $O$-(2-azidoethyl)- $O$-[2-(diglycolylamino)ethyl]heptaethylene glycol, DIPEA, $\mathrm{CH}_{2} \mathrm{Cl}_{2}, 24 \mathrm{~h}$ at r.t. (c) 1-ethynyl-4-(trifluoromethyl)benzene, $\mathrm{CuSO}_{4}$, sodium ascorbate, DMA/water 3:1, $40 \mathrm{~h}$ at r.t.

\subsection{Experimental procedures}

$f$-CNT 1: In a $5 \mathrm{~mL}$ microwave vial were placed glycine $(22 \mathrm{mg}, 0.29 \mathrm{mmol})$, benzaldehyde $(36 \mathrm{mg}, 0.34 \mathrm{mmol})$ and anhydrous dichlorobenzene $(4 \mathrm{~mL})$. The mixture was ultra-sonicated for $\sim 30 \mathrm{~s}$ and the CNT chip was lowered into the solution using a copper clamp on a wire through the septum of a cap. The vial was sealed and the mixture was heated to $\sim 120{ }^{\circ} \mathrm{C}$ under very gentle stirring ( $60 \mathrm{rpm}$ with a small magnetic stirring bar) for $24 \mathrm{~h}$. The mixture was cooled to r.t. and the CNT chip was transferred to a new vial containing $\mathrm{CH}_{2} \mathrm{Cl}_{2}(15 \mathrm{~mL})$ and allowed to stand for $\sim 1 \mathrm{~h}$. This cleaning process was repeated twice and the amine modified CNT forest on chip was dried under ambient conditions and analyzed with SEM equipped with EDS. The EDS data showed a nitrogen peak at $0.39 \mathrm{keV}$. 
$f$-CNT 2: In a $5 \mathrm{~mL}$ microwave vial were placed $O$-(2-azidoethyl)- $O$-[2-(diglycolyl-amino)ethyl]heptaethylene glycol (22 mg, $40 \mu \mathrm{mol})$, PyBOP (23 mg, $44 \mu \mathrm{mol})$ and anhydrous $\mathrm{CH}_{2} \mathrm{Cl}_{2}(5 \mathrm{~mL})$. DIPEA ( $\left.21 \mu \mathrm{L}, 0.120 \mu \mathrm{mol}\right)$ was added and the reaction mixture was stirred at ambient temperature for $1 \mathrm{~h}$. The amine modified CNT forest on chip from the previous step was lowered into the solution using a copper clamp on a wire through the septum of a cap. The vial was sealed and the mixture was gently stirred $(60 \mathrm{rpm})$ at ambient temperature for $24 \mathrm{~h}$. The CNT chip was transferred to a new vial containing $\mathrm{CH}_{2} \mathrm{Cl}_{2}(10 \mathrm{~mL})$ and allowed to stand for $\sim 1 \mathrm{~h}$. This cleaning process was repeated twice and the azide modified CNT forest on chip was dried at ambient conditions and analyzed with SEM equipped with EDS. The EDS data showed a nitrogen peak at $0.39 \mathrm{keV}$.

$f$-CNT 3: In a $5 \mathrm{~mL}$ microwave vial were placed 1-ethynyl-4-(trifluoromethyl)benzene $(16.3 \mu \mathrm{L}, 0.10 \mathrm{mmol})$ and DMA $(3 \mathrm{~mL})$. A solution of sodium ascorbate $(4.5 \mathrm{mg}, 0.023 \mathrm{mmol})$ in water $(0.5 \mathrm{~mL})$ was added followed by a solution of $\mathrm{CuSO}_{4}(0.016 \mathrm{mmol})$ in water $(0.5 \mathrm{~mL})$. The mixture was stirred vigorously for $2 \mathrm{~min}$ and the azide modified CNT forest on chip was lowered into the solution and the reaction mixture was gently stirred $(100 \mathrm{rpm})$ at ambient temperature for $40 \mathrm{~h}$. The CNT chip was transferred to a new vial containing $\mathrm{CH}_{2} \mathrm{Cl}_{2}(15 \mathrm{~mL})$ and allowed to stand for $\sim 2 \mathrm{~h}$. This cleaning process was repeated twice and the triazole modified CNT forest on chip was dried at ambient conditions and analyzed with SEM equipped with EDS. The EDS data showed a fluorine peak at $0.68 \mathrm{keV}$.

$f$-CNT 3 control step: In a $5 \mathrm{~mL}$ microwave vial were placed 1-ethynyl-4-(trifluoromethyl)-benzene $(16.3 \mu \mathrm{L}, 0.10$ mmol) and DMA $(3 \mathrm{~mL})$. A solution of sodium ascorbate $(4.5 \mathrm{mg}, 0.023 \mathrm{mmol})$ in water $(0.5 \mathrm{~mL})$ was added. The mixture was stirred vigorously for $2 \mathrm{~min}$ and the amine modified CNT forest on chip was lowered into the solution and the reaction mixture was gently stirred $(100 \mathrm{rpm})$ at r.t. for $40 \mathrm{~h}$. The CNT chip was transferred to a new vial containing $\mathrm{CH}_{2} \mathrm{Cl}_{2}(15 \mathrm{~mL})$ and allowed to stand for $\sim 2 \mathrm{~h}$. This cleaning process was repeated twice and the unmodified CNT forest on chip was dried at ambient conditions and analyzed with SEM equipped with EDS. The EDS data showed no fluorine peak.

\section{RESULTS}

\subsection{SEM and EDS data}

The modified CNT forests, $f$-CNT 1, $f$-CNT 2, $f$-CNT 3 and $f$-CNT 1 treated with reaction conditions (c) were analyzed using SEM equipped with EDS. The micrograph in Figure 3a shows the CNT structures before functionalization. The EDS spectrum in Figure 3b shows abundant elements on the chip. 


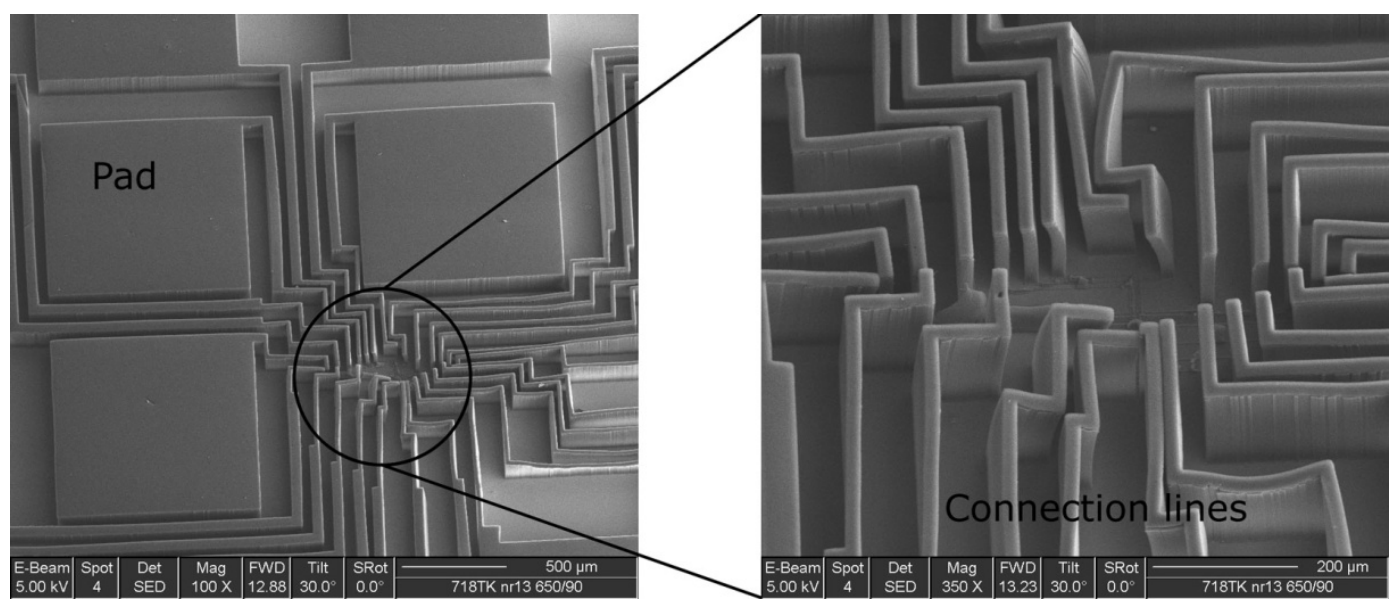

Figure 3a: The SEM micrograph of CNT forests as grown on chip.

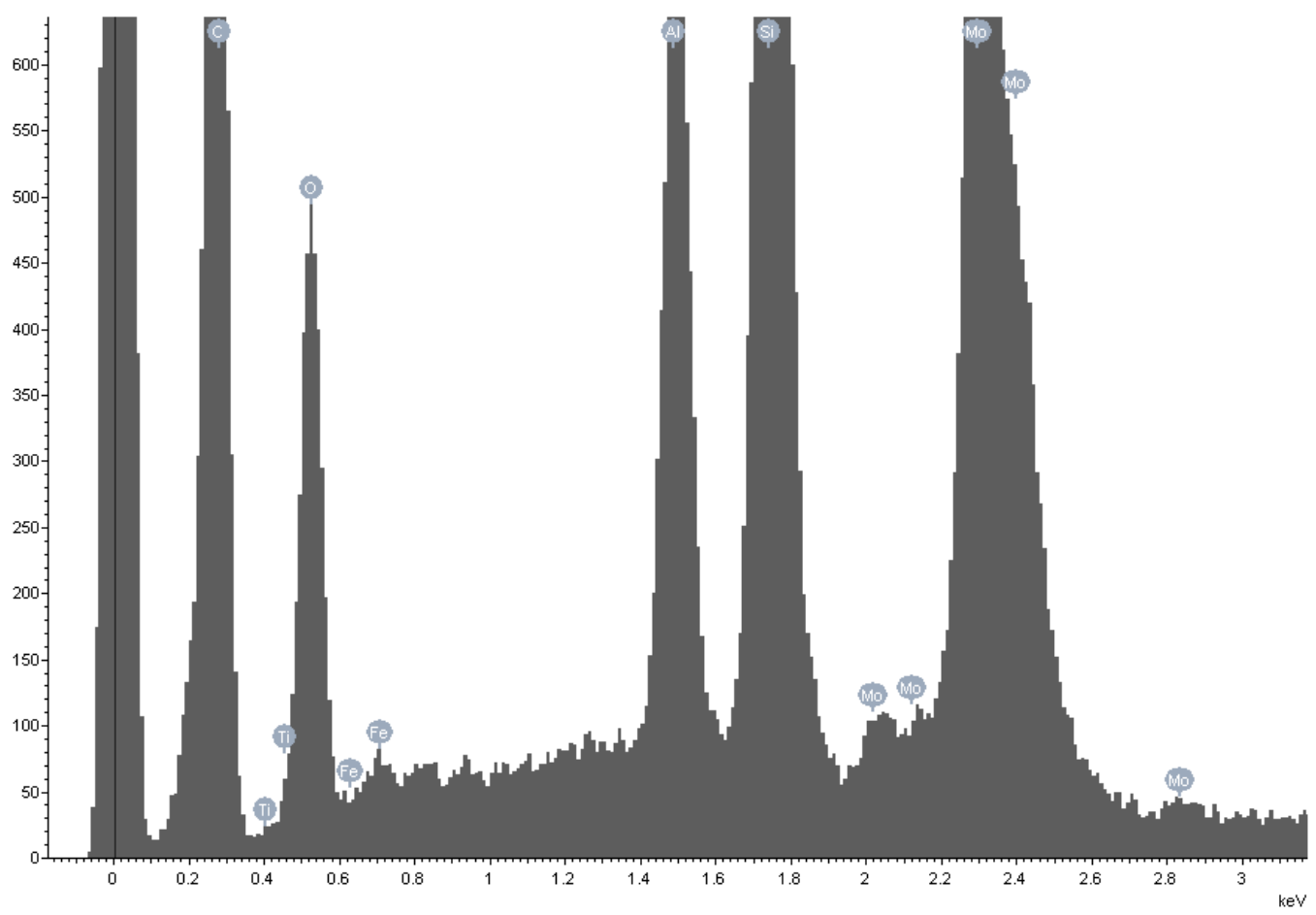

Figure 3b: The EDS spectrum of non functionalized CNT structures. No nitrogen peak is observed.

In Figure $3 \mathrm{~b}$ peaks corresponding to carbon, silicon, oxygen, titanium, molybdenum, aluminum and iron can be seen. They are all consistent with the materials used to fabricate the chip as described in the fabrication process. The SEM micrograph of $f$-CNT $\mathbf{1}$ in Figure 4a shows the CNT structures after the cycloadditon reaction and the EDS spectrum in Figure 4b shows the elements present on the chip. Please note that the CNT forests are still maintaining the morphology of the initial structures as shown in Figure 3a. 


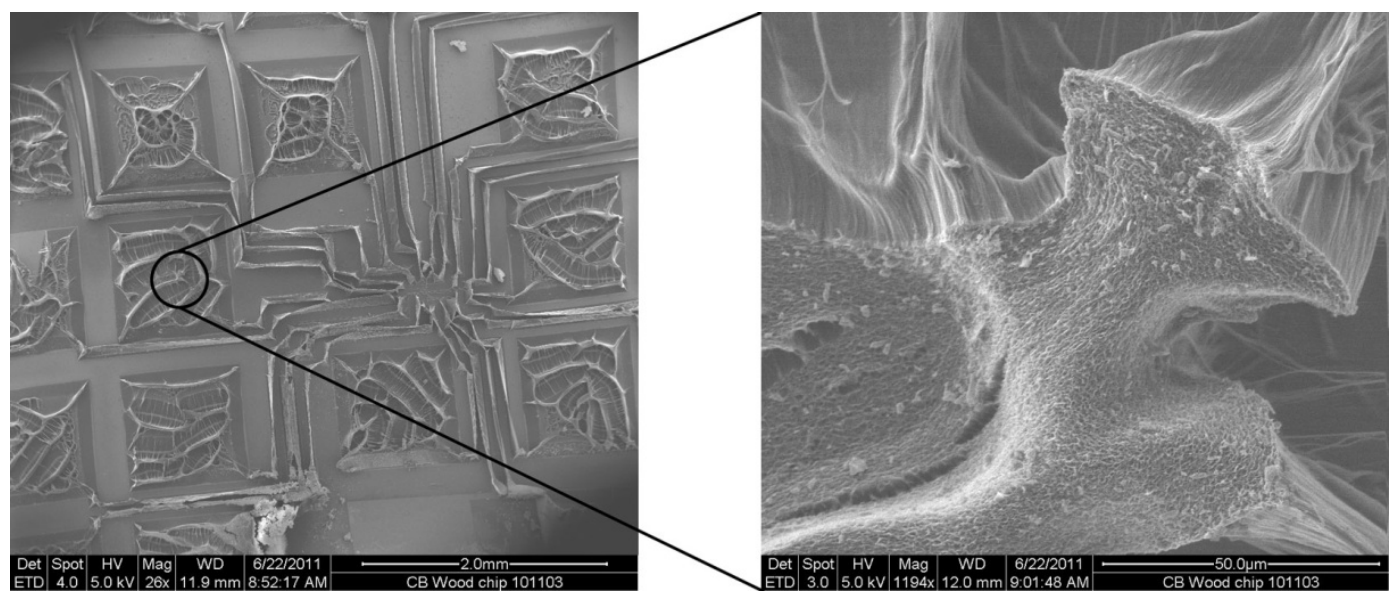

Figure 4a: SEM micrograph of $f$-CNT 1, with an enlargement of the section where EDS was performed.

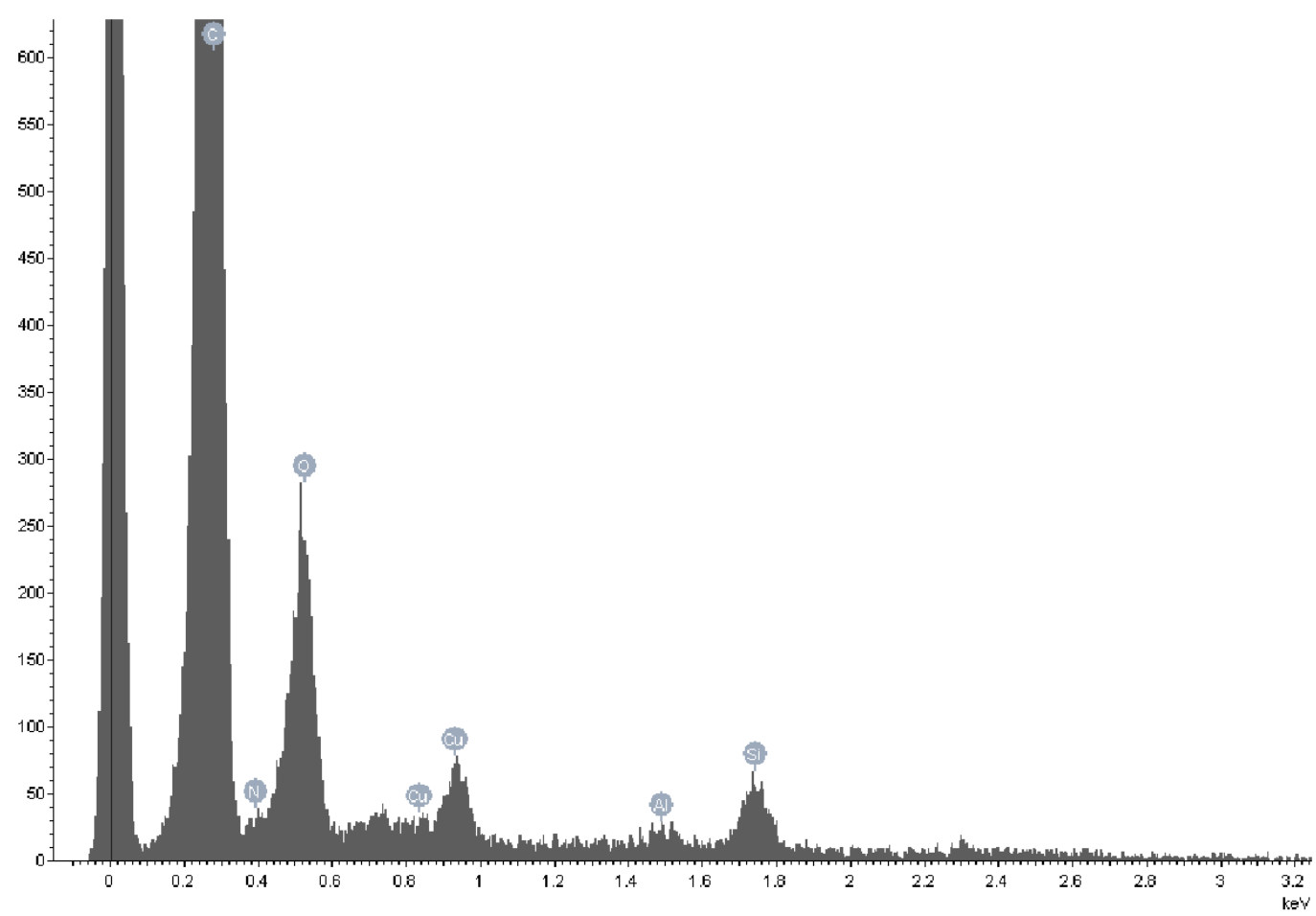

Figure 4b: The EDS spectrum of the $f$-CNT 1 showing a small nitrogen peak at $0.39 \mathrm{keV}$.

The EDS spectra in Figure 4b show a small peak of nitrogen along with the other elements also seen in the EDS spectra of an unmodified chip (Figure 3b). This indicates that the CNTs were functionalized with the cycloaddition product pyrrolidine. We hypothesize that the additional $\mathrm{Cu}$ peak also seen in the spectra, originates from the use of a copper clamp to hold the chip during the reaction conditions (Figure 2). The same sequence of SEM and EDS was repeated with the $f$-CNT 2 chip with azide functional groups attached to the CNT structures and the results are shown in Figure 5a-b. 


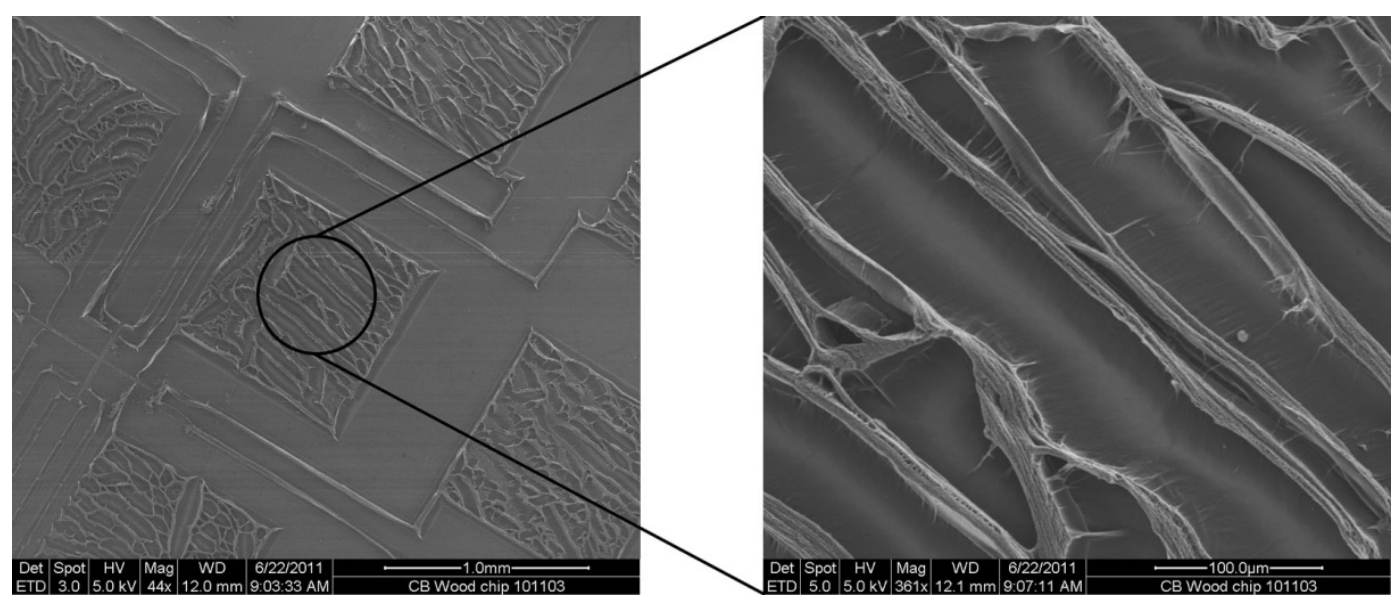

Figure 5a: SEM micrograph of the section of CNT forest where the EDS was performed on $f$-CNT 2.

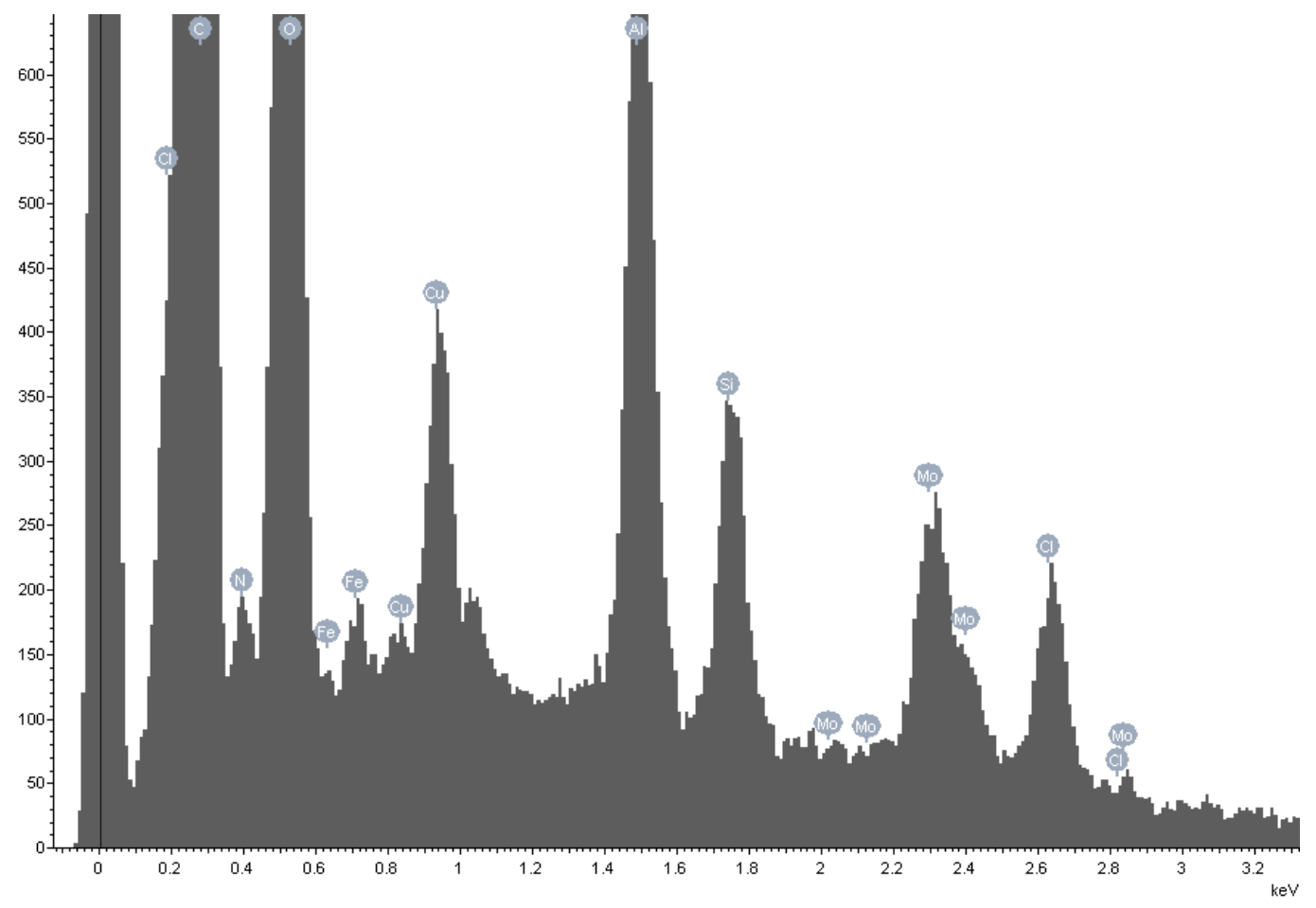

Figure 5b: The EDS spectrum of $f$-CNT 2 shows a clear nitrogen peak at $0.39 \mathrm{keV}$.

The nitrogen peak in the spectrum of $f$-CNT 2 (Figure $5 \mathrm{~b}$ ) is a little stronger than that in $f$-CNT 1 (Figure $4 \mathrm{~b}$ ) as more nitrogen is added to the CNT structures after the second reaction step. Since the difference between the two EDS spectra in Figure 4 and 5 is not large enough to give a satisfactory confirmation of the second reaction, a third reaction was performed in order to introduce a new element onto the CNT forests on-chip. A CuAAC reaction was performed to 
introduce fluorine that can be easily detected in EDS spectra. Figure 6 show the SEM micrograph and the EDS spectrum of $f$-CNT 3. An EDS spectrum was also recorded in an area of the chip without any CNTs, are shown in Figure 7.

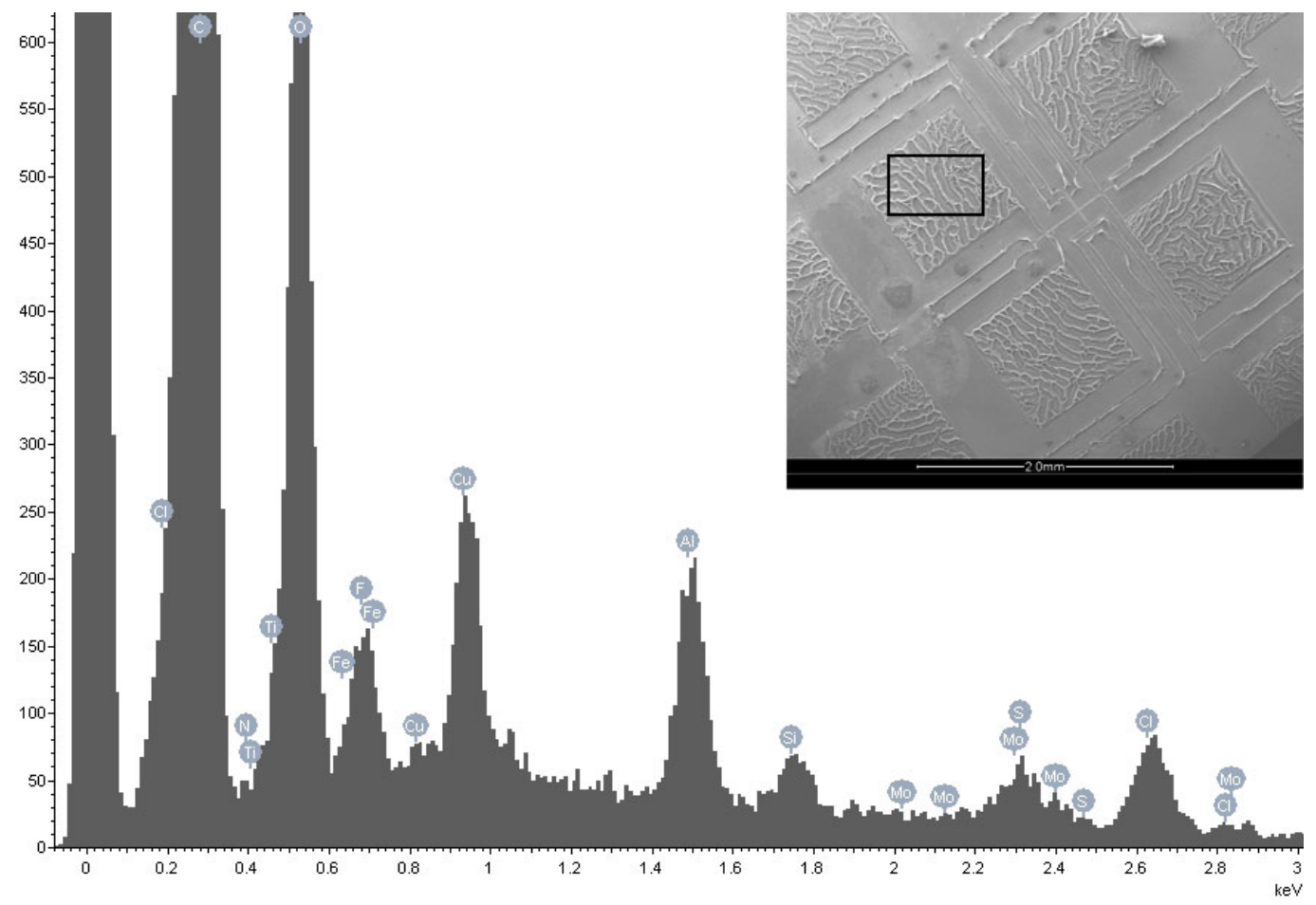

Figure 6. SEM micrograph of the section of CNT forest where EDS was performed on $f$-CNT 3.

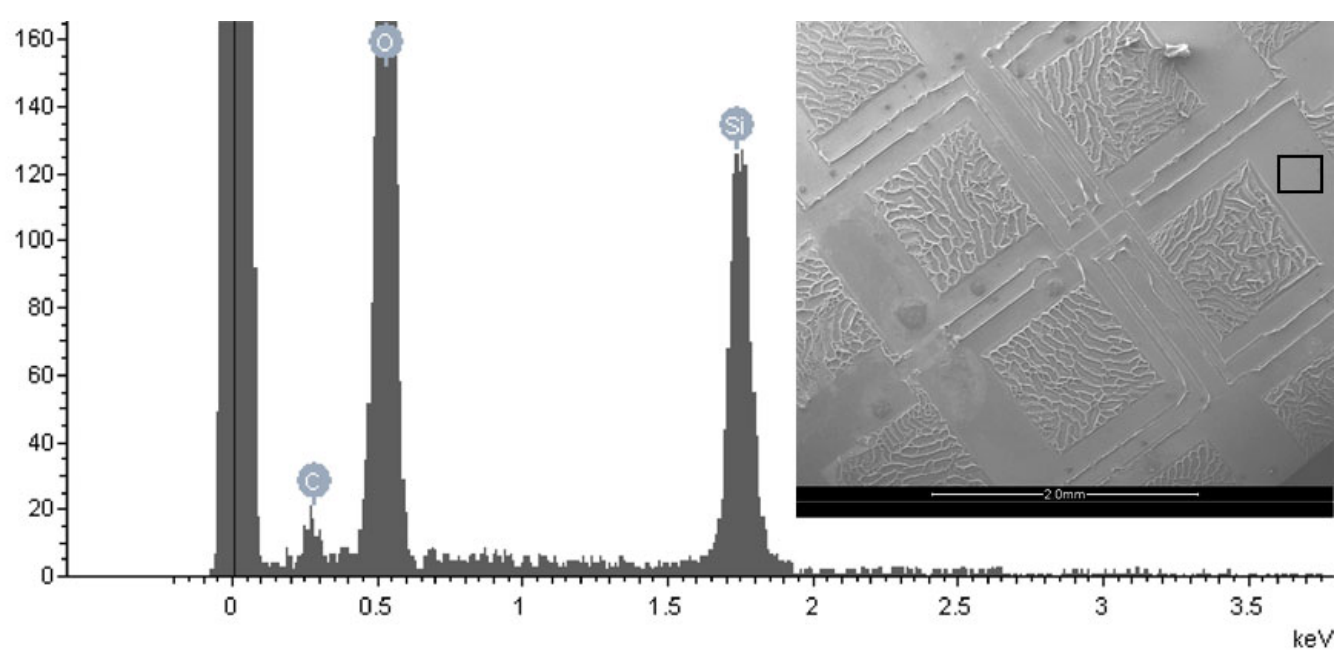

Figure 7. SEM micrograph of a section with no CNT forests, where the EDS was performed on $f$-CNT 3. 
As a control that the fluorine peak in the EDS spectra does not originate from non-covalent deposition of 1-ethynyl-4(trifluoromethyl)benzene on the CNT forests, $f$-CNT 1 was also treated under similar reaction conditions as $f$-CNT 2 above. Figure 8 shows the SEM micrograph and the EDS spectra of the control reaction.

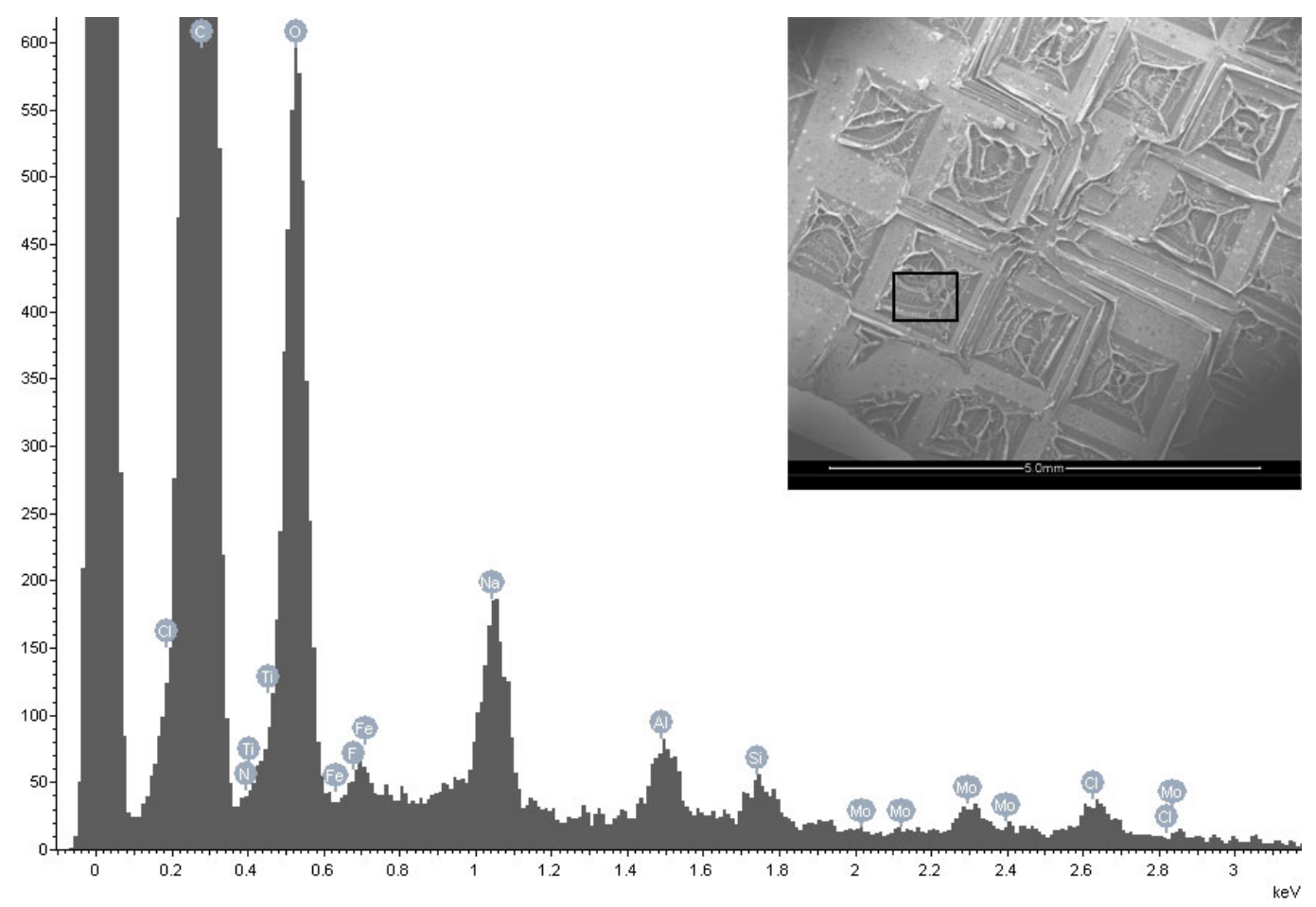

Figure 8. SEM micrograph of the section of CNT forest where EDS was performed on the control chip.

According to the EDS spectrum in Figure 6, the fluorine peak at $0.68 \mathrm{keV}$ indicates that the CNT forests are covalently functionalized with fluorine. The EDS spectrum of the control experiment (Figure 8) shows no clear fluorine peak, but only the iron peak nearby. These results together indicate that the fluorine atoms in $f$-CNT 3 are covalently attached. This also confirms that $f$-CNT 2 was successfully functionalized with active azide groups. The small peak at $0.70 \mathrm{keV}$ in Figure 8 most likely originates from iron. However, this may be interpreted as some non-covalent binding of the fluorine molecules with the CNTs being present and that the fluorine peak overlaps with the iron peak nearby in the EDS spectra.

\subsection{Discussion}

The spectrum in Figure $3 \mathrm{~b}$ is from a CNT chip that has Ti/Mo as the metal film base (to get contact between the CNTs for conductivity) but the CNTs are not functionalized in this case. The EDS spectrum of $f$-CNT 1 (Figure $4 \mathrm{~b}$ ) and $f$-CNT 2 (Figure 5b) are obtained from similar chips after step 1 and step 2 functionalization, respectively. The nitrogen peaks in the latter spectra provide evidence that CNT structures are indeed functionalized by the reactions carried out. Interestingly, the morphology of the CNT structures is not significantly affected and the CNT structures are still electrically conducting. To further validate the $f$-CNT 2 structure, a CuAAC reaction was performed to obtain $f$-CNT 3, which was confirmed by a fluorine peak at $0.68 \mathrm{keV}$ (Figure 6). As a control reaction, $f$-CNT 1 was treated under similar conditions as $f$-CNT 2 and no fluorine peak was observed in the EDS spectra (Figure 8). 
A comparison of the morphology of the CNT structures on the chips with and without functionalization of CNT forests is also presented. As seen in the SEM micrographs (Figures 4-6) the connection lines to the pads are well defined even after the functionalization of CNT structures. On the other hand, the pad structures seem somehow worn down and warped. The reason of such contrast in the morphology of CNT structures lies in the aspect ratio (the area of the catalyst on substrate. i.e. the base of the CNT forest and the length of CNTs). Since, the aspect ratio for the pads is almost a reciprocal of the aspect ratio for the contact lines, the CNTs clamp into dense sections where they can achieve the least energy structures when the solvent evaporates. The capillary forces acting on the CNTs force them to "bunch up" and form packs of dense structures. By way of contrast, the base area compared to the height of the CNTs for the contact lines is much smaller. Hence, CNTs appear to form a wall like structure, indicating that we can control the resolution of the structure (almost 5 times smaller compared to the original) once the CNT forest structures is exposed to a solvent and subsequent evaporation.

\section{CONCLUSIONS}

In the EDS data on $f$-CNT 2 , the presence of nitrogen indicates that we have successfully attached the azide group on $f$ CNT 2 by the two-step reaction scheme described in Scheme 2. However, since EDS does not provide quantitative data, a third reaction was carried out to verify the successful synthesis of the $f$-CNT 2 structure. By performing a CuAAC reaction on $f$-CNT 2 with a fluorine source, $f$-CNT 3 was successfully synthesized. The fluorine peak in the EDS spectra (Figure 6.) of $f$-CNT 3 together with the absence of fluorine in the control experiment, confirms successful chemical reaction on the CNTs. This result also demonstrates that the azide functional group can be used to attach other interesting molecules to the CNT structure as they are grown on chip. Thus, the qualitative information from the EDS spectra above clearly justifies our conclusion that we have successfully achieved covalent functionalization of surface-grown CNTs on metal films as the contact metal layer.

Importantly, as concluded from the EM data, this technique does not significantly affect the morphology of the CNT forests, but the structures are preserved and, hence, may be exploited, for example, as arrays of sensing surfaces.

\section{ACKNOWLEDGEMENT}

The CNT forests were fabricated using the MC2 Nanofabrication facilities at Chalmers University of Technology. The SEM EDS was performed at the department of Applied Physics at Chalmers with help from Anders Kvist. The research was funded by grants to B.N. from the European Research Council (ERC) and King Abdullah University of Science and Technology (KAUST).

\section{REFERENCES}

[1] Balasubramanian K. and Burghard M., "Chemically Functionalized Carbon Nanotubes", Small 1 (2), 180-192 (2005).

[2] Qureshi A., Kang W. P., Davidson J. L. and Gurbuz Y., "Review on carbon-derived, solid-state, micro and nano sensors for electrochemical sensing applications", Diamond and Related Materials 18, 1401-1420 (2009).

[3] Chen H., Roy A., Baek JB., Zhu L., Qu J. and Dai L., "Controlled growth and modification of vertically-aligned carbon nanotubes for multifunctional applications", Materials Science and Engineering R 70, 63-91 (2010). 
[4] Yang W., Thordarson P., Gooding J. J., Ringer S. P. and Braet F., "Carbon nanotubes for biological and biomedical applications", Nanotechnology 18 (41), 2001 (2007).

[5] Qiao R. and Ke P.C., "Lipid-Carbon Nanotube Self-Assembly in Aqueous Solution”, J. Am. Chem. Soc. 128, 1365613657 (2006).

[6] Wallace E. J. and Sansom M. S. P., "Carbon nanotube self-assembly with lipids and detergent: a molecular dynamics study”, Nanotechnology 20 (04), 5101 (2009).

[7] Tran P. D., Le Goff A., Heidkamp J., Jousselme B., Guillet N., Palacin S., Dau H., Fontecave M., and Artero V., "Noncovalent Modification of Carbon Nanotubes with Pyrene-Functionalized Nickel Complexes: Carbon Monoxide Tolerant Catalysts for Hydrogen Evolution and Uptake”, Angew. Chem. Int. Ed. 50, 1371-1374 (2011).

[8] Oelsner C., Herrero M. A., Ehli C., Prato M., and Guldi D. M., "Charge Transfer Events in Semiconducting SingleWall Carbon Nanotubes”, J. Am. Chem. Soc. 133 (46), 18696-18706 (2011).

[9] Georgakilas V., Tagmatarchis N., Pantarotto D., Bianco A., JP. Briand JP. and Prato M., "Amino acid functionalisation of water soluble carbon nanotubes", Chem. Commun., 3050-3051 (2002).

[10] Maggini, M., Scorrano, G., Prato, M., “Addition of azomethine ylides to $\mathrm{C}_{60}$ : synthesis, characterization, and functionalization of fullerene pyrrolidines”, J. Am. Chem. Soc 115, 9798-9799 (1993).

[11] Georgakilas, V., Kordatos, K, Prato, M., Guldi, D. M., Holzinger, M., Hirsch A., "Organic Functionalization of Carbon Nanotubes", J. Am. Chem. Soc. 124, 760-761 (2002).

[12] Coste, J., Le-Nguyen, D., Castro, B., "РyBOP®: A new peptide coupling reagent devoid of toxic by-product", Tetrahedron Lett. 31, 205-208 (1990).

[13] Tornoe, C. W., Christensen, C., Meldal, M., "Peptidotriazoles on Solid Phase: [1,2,3]-Triazoles by Regiospecific Copper(I)-Catalyzed 1,3-Dipolar Cycloadditions of Terminal Alkynes to Azides”, J. Org. Chem. 67, 3057 (2002).

[14] Rostovtsev V. V., Green L. G., Fokin V. V., and Sharpless K. B., "A Stepwise Huisgen Cycloaddition Process: Copper(I)-Catalyzed Regioselective "Ligation" of Azides and Terminal Alkynes", Angew. Chem. Int. Ed. 41 (14), 25962599 (2002). 